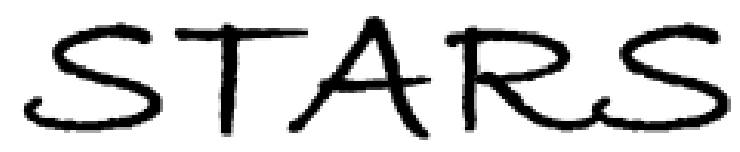

University of Central Florida

STARS

$1-1-2008$

\title{
Single-photon Mach-Zehnder interferometer for quantum networks based on the single-photon Faraday effect
}

\author{
H. P. Seigneur \\ University of Central Florida \\ Michael N. Leuenberger \\ University of Central Florida \\ W. V. Schoenfeld \\ University of Central Florida
}

Find similar works at: https://stars.library.ucf.edu/facultybib2000

University of Central Florida Libraries http://library.ucf.edu

This Article is brought to you for free and open access by the Faculty Bibliography at STARS. It has been accepted for inclusion in Faculty Bibliography 2000s by an authorized administrator of STARS. For more information, please contact STARS@ucf.edu.

\section{Recommended Citation}

Seigneur, H. P.; Leuenberger, Michael N.; and Schoenfeld, W. V., "Single-photon Mach-Zehnder interferometer for quantum networks based on the single-photon Faraday effect" (2008). Faculty Bibliography 2000s. 956.

https://stars.library.ucf.edu/facultybib2000/956

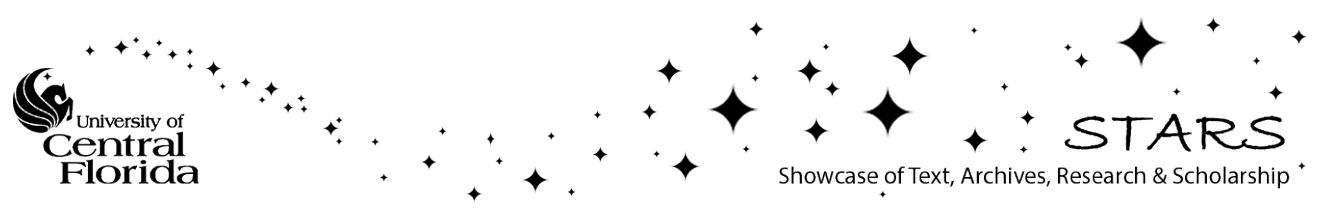




\section{Single-photon Mach-Zehnder interferometer for quantum networks based on the single- photon Faraday effect}

Cite as: J. Appl. Phys. 104, 014307 (2008); https://doi.org/10.1063/1.2948924

Submitted: 31 December 2007 . Accepted: 21 April 2008. Published Online: 09 July 2008

H. P. Seigneur, Michael N. Leuenberger, and W. V. Schoenfeld

\section{ARTICLES YOU MAY BE INTERESTED IN}

Invited Review Article: Single-photon sources and detectors

Review of Scientific Instruments 82, 071101 (2011); https://doi.org/10.1063/1.3610677

A hands-on introduction to single photons and quantum mechanics for undergraduates

American Journal of Physics 78, 471 (2010); https://doi.org/10.1119/1.3354986

General properties of lossless beam splitters in interferometry

American Journal of Physics 49, 882 (1981); https://doi.org/10.1119/1.12387

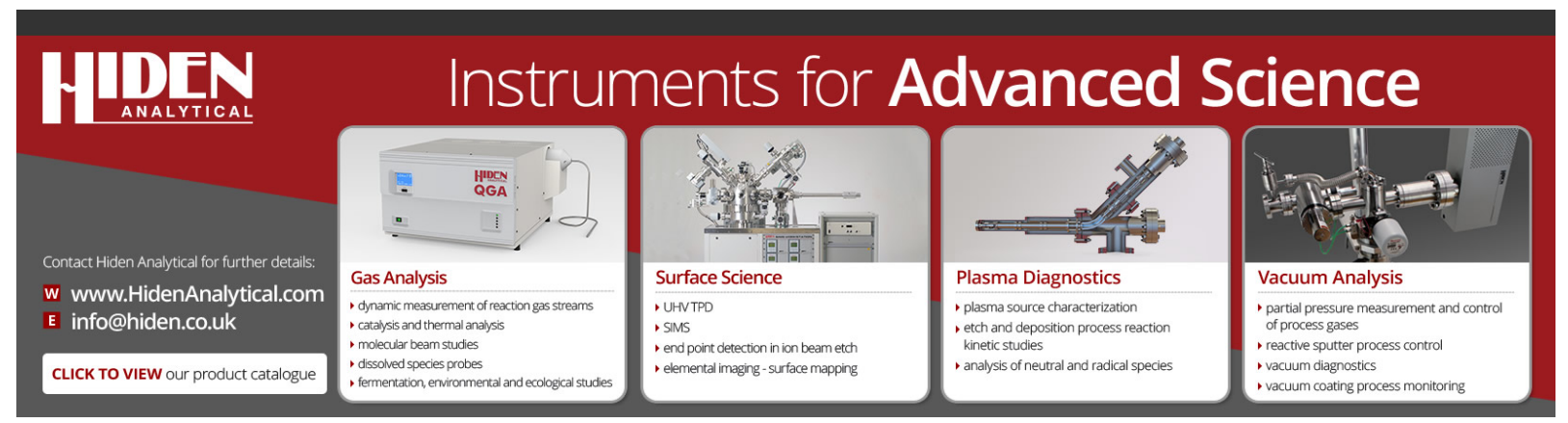




\title{
Single-photon Mach-Zehnder interferometer for quantum networks based on the single-photon Faraday effect
}

\author{
H. P. Seigneur, ${ }^{1}$ Michael N. Leuenberger, ${ }^{2, a)}$ and W. V. Schoenfeld ${ }^{1, b)}$ \\ ${ }^{1}$ College of Optics and Photonics (CREOL), University of Central Florida, Orlando, Florida 32816, USA \\ ${ }^{2}$ NanoScience Technology Center and Department of Physics, University of Central Florida, Orlando, \\ Florida 32826, USA
}

(Received 31 December 2007; accepted 21 April 2008; published online 9 July 2008)

\begin{abstract}
Combining the recent progress in semiconductor nanostructures along with the versatility of photonic crystals in confining and manipulating light, quantum networks allow for the prospect of an integrated and low power quantum technology. Within quantum networks, which consist of a system of waveguides and nanocavities with embedded quantum dots, it has been demonstrated in theory that many-qubit states stored in electron spins could be teleported from one quantum dot to another via a single photon using the single-photon Faraday effect. However, in addition to being able to transfer quantum information from one location to another, quantum networks need added functionality such as (1) controlling the flow of the quantum information and (2) performing specific operations on qubits that can be easily integrated. In this paper, we show how a single-photon Mach-Zehnder interferometer (SMZI), that uses the concept of the single-photon Faraday effect to manipulate the polarization of a single photon, can be operated both as a switch to control the flow of quantum information inside the quantum network and as various single-qubit quantum gates to perform operations on a single photon. Given that the $X$ gate, the $Z$ gate, and the $X Z$ gate are essential for the implementation of quantum teleportation, we show explicitly their implementation by means of our proposed SMZI. We also present the implementation of the Hadamard gate and the single-qubit phase gate, which are needed to complete the universal set of quantum gates for integrated quantum computing in a quantum network. Finally, the expected fidelity and robustness of the proposed SMZI are quantitatively explored by considering the phase errors within the SMZI.

(C) 2008 American Institute of Physics. [DOI: 10.1063/1.2948924]
\end{abstract}

\section{INTRODUCTION}

Single-photon interferometry has been, and continues to be, a valuable method to study and understand a vast range of phenomena in physics, particularly in quantum optics such as quantum superposition," the "quantum eraser," which is the possibility of choosing the determination of paths after the interferometer, ${ }^{2}$ dephasing due to the quantum channel resulting from the inevitable interaction between the channel and the quanta of light, ${ }^{3}$ and even the quantum Sagnac effect. ${ }^{4}$ In addition, single-photon interferometers can also play a key role as core components of new devices in the field of quantum information in a similar way that integrated Mach-Zehnder (MZ) interferometers are fundamental elements of photonic switches in optical communication. In fact, Shimizu and Imoto $^{5}$ have already proposed a singlephoton interferometer for cryptographic quantum communication. Rather than manipulating and measuring the internal states of entangled photon twins, cryptographic quantum communication is possible by manipulating a single photon in an extended interferometer. Knill et al. ${ }^{6}$ showed that efficient quantum computation is possible using only linear optical components (beam splitters and phase shifters, i.e., elements that make up MZ interferometers), single-photon sources, and photodetectors. Although the use of single-

\footnotetext{
${ }^{\text {a) }}$ Electronic mail: mleuenbe@mail.ucf.edu.

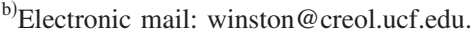

photon interferometers has been verified in processing quantum information in the case of quantum key distribution ${ }^{5}$ and, in principle, in quantum computation, ${ }^{6}$ they have been typically implemented in a way that was relatively bulky and inadequate for use within a high density integration setting. Yet, there is much interest in a quantum technology that can be implemented on a chip. One important reason is that an integrated quantum technology could in theory continue to fulfill Moore's law simply by adding qubits to the Hilbert space, ${ }^{7}$ and as a result, constitute itself as a potentially attractive solution to the current difficult challenges of further decreasing transistor size in order to fulfill Moore's law, assuming the cost of fabricating such quantum technology can be kept reasonable.

The approach we examine in this paper is based on quantum networks, ${ }^{8}$ which are deemed very promising given recent progresses in the conception and fabrication of semiconductor nanostructures and photonic crystals, along with single-photon on-demand sources. One important implementation of quantum networks permits the teleportation of quantum information from qubits made of the spin of an excess electron in the conduction band of quantum dots (QDs) embedded in nanocavities that interact via single photons by means of the single-photon Faraday effect (SPFE). ${ }^{9}$ Such spin-based implementation of a quantum network allows for the prospect of an integrated and low power ${ }^{10}$ quan- 
tum technology in which single-photon MZ interferometers (SMZIs) are envisioned to play a fundamental role.

In this paper, the operating principle and properties of SMZIs and their applications are introduced. In Sec. II we provide an overview of the SPFE, the fundamental basis that enables the operation of SMZIs. By means of this effect, SMZIs are able to manipulate the geometrical phase of a single photon as a switching mechanism in order to control the flow of quantum information within a quantum network. Using the geometrical phase instead of an optical path difference within a MZ interferometer is a bit unusual, however, it allows for an additional functionality-it can also be used to create a superposition of the photon polarization eigenbasis or simply a change of eigenbasis (i.e., linear to circularly polarized light). In other words, SMZIs could also be used as single-qubit gates that are critical components of quantum networks in which single photons and their polarization eigenbasis are used to realize "messenger" qubits. In Sec. III, these two separate applications of SMZIs, namely, an optical switch and a single-qubit gate, are discussed in detail. Design issues, dynamic behavior, as well as the anticipated performance and fidelity of SMZIs are presented in Sec. IV.

\section{THEORETICAL BASIS OF THE SPFE}

The classical Faraday effect is a linear magneto-optic effect, which is characterized by a rotation of the linear polarization of light propagating inside an isotropic medium subject to an external constant magnetic field applied in the direction of propagation. The general concept behind the Faraday effect is that a linearly polarized wave can be decomposed into two circularly polarized waves, which are the appropriate normal modes in this regime; each circularly polarized normal mode propagates with different refractive indices. Quantum mechanics tells us that the magnetic field induced splitting of the energy levels with different total angular momenta is the reason for the linear polarization rotation, and hence the different circular polarizations of light couple differently during the process of virtual absorption, which is responsible for the existence of refractive indices. Similarly, the SPFE involves the rotation of linearly polarized light as a result of broken symmetry between the left and right components of circularly polarized light. However, the SPFE only involves the nonresonant interaction of a single photon with a two-level system, does not require an external magnetic field, and can result in an entanglement of the photon with an electron spin. ${ }^{9}$

The two-level system of interest, as depicted in Fig. 1, can be deduced under certain assumptions. First, the split-off band is purposely ignored since typical split-off energies are on the order of several hundreds of $\mathrm{meV}$, thus bringing the energy level well out of resonance with the single photon. Second, spherical dots are assumed in order to maintain a twofold energy degeneracy in the conduction band and a fourfold energy degeneracy in the valence band at the $\Gamma$ point. Also, due to quantum size effects within the QD, the effective band gap is shifted to higher energies. Third, under the appropriate extrinsic doping and thermal conditions, it can be assumed that the top of the valence band is filled with

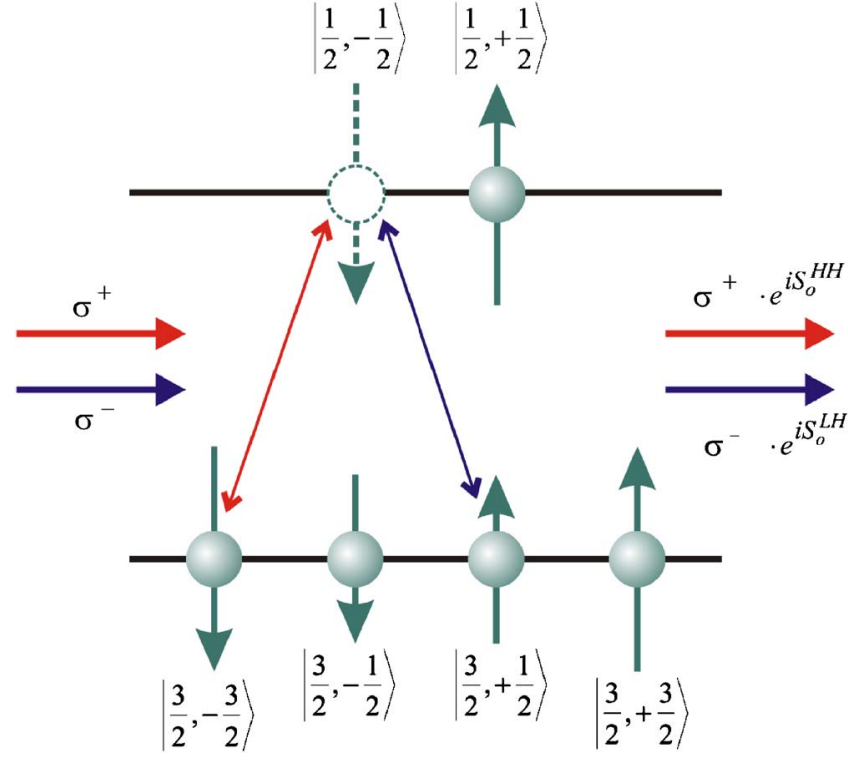

FIG. 1. (Color online) Two-level system of a QD, depicting the SPFE. The rotation of the single-photon linear polarization is clockwise in the plane perpendicular to its propagation when the excess electron spin is up ( $\uparrow)$.

four electrons, while there is an excess electron in the conduction band. It is the presence of this extra conduction band electron that enables the SPFE. If the energy of the single photon is taken to be slightly below the effective band gap energy (i.e., slightly detuned), considering the parity condition imposed from the matrix element of envelop functions in semiconductor nanostructures, the transition from the top of the valence band $(m=1)$ to the bottom of the conduction band $(n=1)$ is the strongest transition by far. Since the matrix elements of other transitions are much weaker, one can then consider this transition as a two-level system.

We now isolate our consideration to this two-level system. Recalling that the single-photon energy is slightly detuned from the two-level system resonance, the SPFE is a virtual process resulting from the transition rules that govern the electric-dipole interaction. In fact, it is the transition rules that allow the symmetry of the circularly polarized light to be broken as it couples to the two-level system during propagation, resulting in the rotation of the single-photon polarization. This is much different than the shift in energy level due to an external magnetic field exploited in the classical case. The ensuing rotation of the polarization is a consequence of one circular polarization, say right-hand circular polarization (RCP), interacting with only the heavy hole band and the other circular polarization, left-hand circular polarization (LCP) with the light hole band as depicted in Fig. 1. Since the matrix element involving the heavy hole band is larger than the matrix element involving the light hole band, both circularly polarized components accumulate different phases, resulting in a rotation of the linear polarization. Using the Jaynes-Cummings model, the Hamiltonian of the system can be written as

$$
\begin{aligned}
H= & \hbar \omega_{\mathrm{cav}}\left(a_{\sigma^{+}}^{\dagger} a_{\sigma^{+}}+a_{\sigma^{-}}^{\dagger} a_{\sigma^{-}}\right)+\hbar \omega_{h h} \sigma_{3 / 2 v}+\hbar \omega_{h h} \sigma_{-3 / 2 v} \\
& +\hbar \omega_{l h} \sigma_{1 / 2 v}+\hbar \omega_{l h} \sigma_{-1 / 2 v}+\hbar \omega_{e} \sigma_{1 / 2 c}+\hbar \omega_{e} \sigma_{-1 / 2 c} \\
& +\hbar g_{3 / 2 v, 1 / 2 c}\left(a_{\sigma^{-}}^{\dagger} \sigma_{3 / 2 v, 1 / 2 c}+a_{\sigma^{-}} \sigma_{1 / 2 c, 3 / 2 v}\right.
\end{aligned}
$$




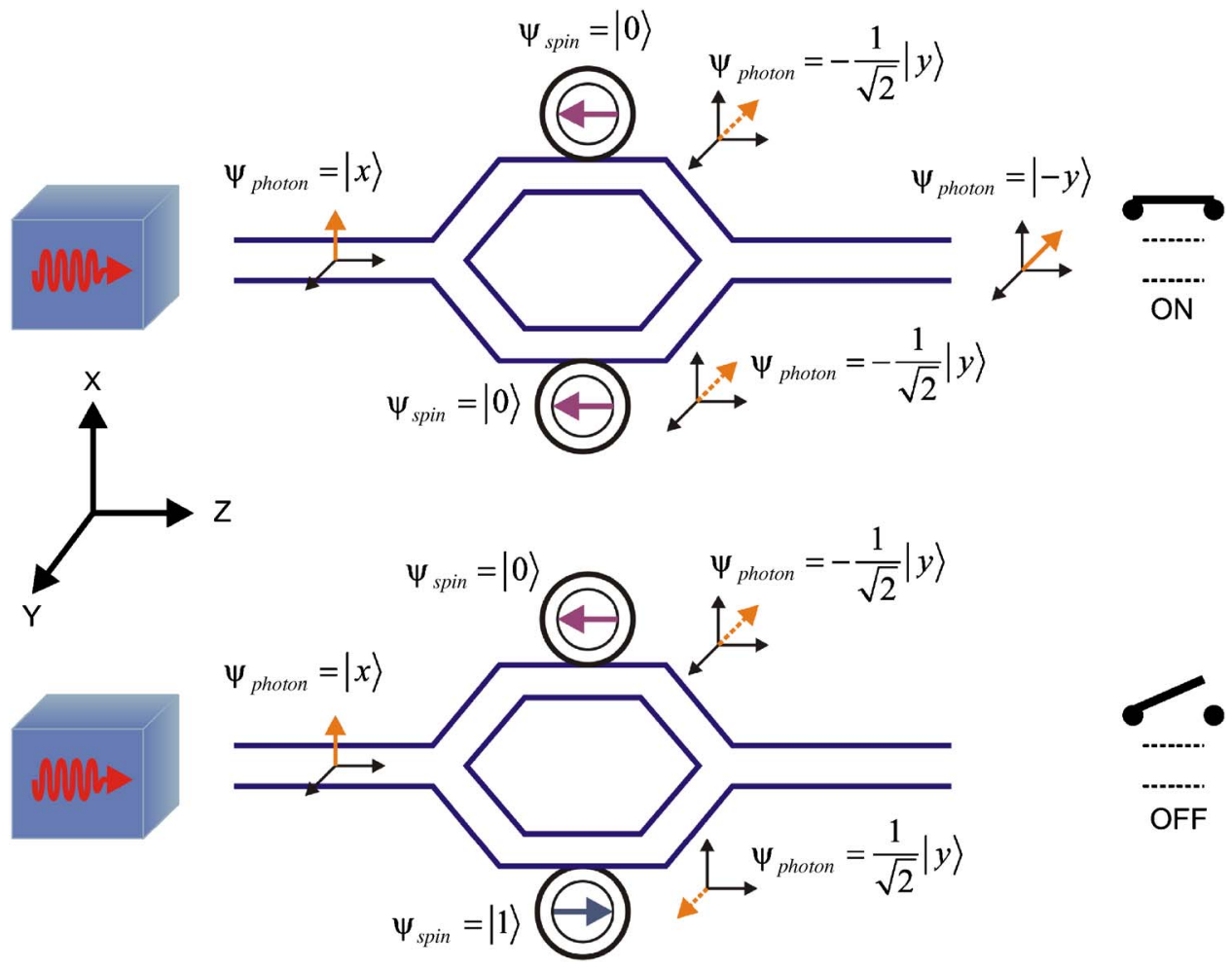

FIG. 2. (Color online) Schematic for a spin-based SMZI where the spins of the electrons in the upper and lower arms are either parallel (above) or antiparallel (below), giving, respectively, an identical and an opposite rotation of the polarization.

$$
\begin{aligned}
& \left.+a_{\sigma^{+}}^{\dagger} \sigma_{-3 / 2 v,-1 / 2 c}+a_{\sigma^{+}} \sigma_{-1 / 2 c,-3 / 2 v}\right) \\
& +\hbar g_{1 / 2 v, 1 / 2 c}\left(a_{\sigma^{-}}^{\dagger} \sigma_{1 / 2 v,-1 / 2 c}+a_{\sigma^{-}} \sigma_{-1 / 2 c, 1 / 2 v}\right. \\
& \left.+a_{\sigma^{+}}^{\dagger} \sigma_{-1 / 2 v, 1 / 2 c}+a_{\sigma^{+}} \sigma_{1 / 2 c,-1 / 2 v}\right)
\end{aligned}
$$

where

$$
\begin{aligned}
H_{\text {field }}= & \hbar \omega_{\text {cav }}\left(a_{\sigma^{+}}^{\dagger} a_{\sigma^{+}}+a_{\sigma^{-}}^{\dagger} a_{\sigma^{-}}\right) \\
H_{\text {atom }}= & \hbar \omega_{h h 1,2} \sigma_{3 / 2 v}+\hbar \omega_{h h 1,2} \sigma_{-3 / 2 v}+\hbar \omega_{l h 1,2} \sigma_{1 / 2 v} \\
& +\hbar \omega_{l h 1,2} \sigma_{-1 / 2 v}+\hbar \omega_{e 1,2} \sigma_{1 / 2 c}+\hbar \omega_{e 1,2} \sigma_{-1 / 2 c} \\
H_{J-C}= & \hbar g_{3 / 2 v, 1 / 2 c}\left(a_{\sigma^{-}}^{\dagger} \sigma_{3 / 2 v, 1 / 2 c}+a_{\sigma^{-}} \sigma_{1 / 2 c, 3 / 2 v}\right. \\
& \left.+a_{\sigma^{+}}^{\dagger} \sigma_{-3 / 2 v,-1 / 2 c}+a_{\sigma^{+}} \sigma_{-1 / 2 c,-3 / 2 v}\right) \\
& +\hbar g_{1 / 2 v, 1 / 2 c}\left(a_{\sigma^{-}}^{\dagger} \sigma_{1 / 2 v,-1 / 2 c}+a_{\sigma^{-}} \sigma_{-1 / 2 c, 1 / 2 v}\right. \\
& \left.+a_{\sigma^{+}}^{\dagger} \sigma_{-1 / 2 v, 1 / 2 c}+a_{\sigma^{+}} \sigma_{1 / 2 c,-1 / 2 v}\right)
\end{aligned}
$$

Figure 1 shows how different circularly polarized light interacts with the two-level system. The direction of the singlephoton Faraday rotation is conditional on the spin orientation of the excess electron, i.e., it is clockwise if the spin is up ( $\uparrow)$ as depicted in Fig. 1, and counterclockwise if the spin is down $(\downarrow)$. The single-photon Faraday rotation is a result of the different phases accumulated for the right circularly polarized $\left(\sigma^{+}\right)$and the left circularly polarized $\left(\sigma^{-}\right)$light during interaction with the two-level system or QD. ${ }^{9}$

\section{APPLICATIONS}

Interferometers usually are composed of two basic elements: (1) passive structural components for splitting and recombining light and (2) active components for manipulat- ing the phase of light. First, passive structural components for splitting and recombining the light considered in this paper are based on photonic crystals (see Sec IV for details on design issues). In the case of the switch, the amplitude of the single-photon field is split in half; whereas, in the case of the single-qubit gate, the single-photon field is split according to its polarization. Second, the active components in a SMZI consist of a pair of nanocavities, each coupled to one arm of the SMZI. Each cavity and its embedded QD provide the environment for the SPFE to take place and therefore provide the means for the manipulation of the photon polarization. Upon completion of the desired amount of rotation of the linear polarization inside the nanocavities, the photon is released into the respective arms of the SMZI.

\section{A. SMZ interferometer as a switch}

Figure 2 shows conceptually how the SMZI can be used as a switch. Contrary to the approach used in Ref. 9 in which the excess electron spin in the QDs within nanocavities is initialized to be in a superposition of up/down states for the purpose of entangling it with the single photon; here the spin is either up or down (in a pure state). There exists a variety of optical, electrical, and magnetic techniques that can be used to initialize spins in semiconductors. ${ }^{11}$ As for the quantization axis of the spins, it must be along the direction of propagation of the single photon.

In its on configuration (upper portion of Fig. 2), an initially $x$ polarized (out of plane) photon is split into both arms of the SMZI, then in each arm the single photon couples into nanocavities and interacts by means of the SPFE [strong coupling regime $(\mathrm{SCR})]$ with QDs that have their excess electron spin initialized in the down position or in the $-z$ direction (in plane). This causes its polarization to be rotated in 


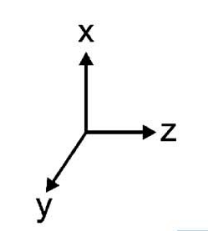

\section{Control}

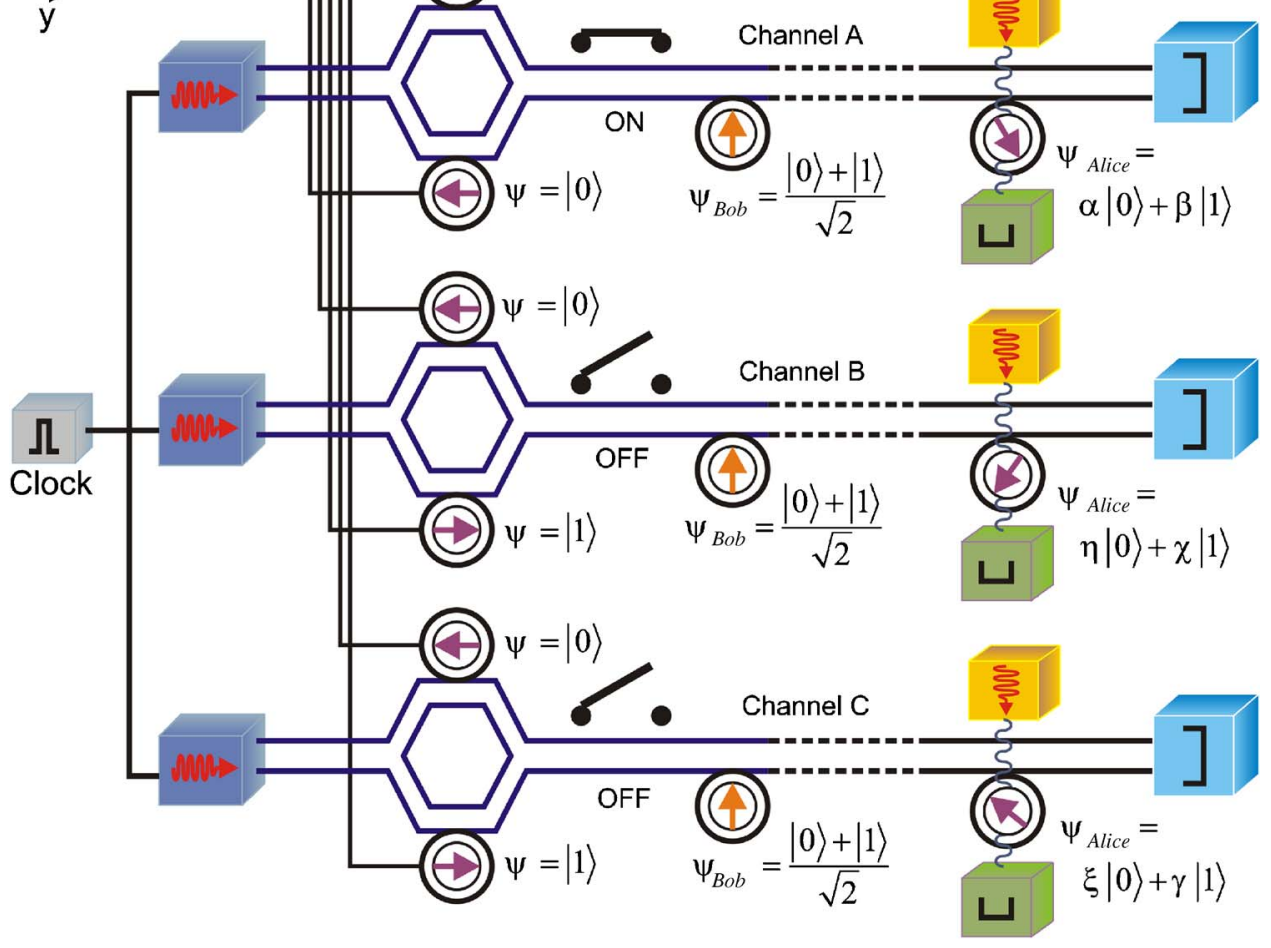

FIG. 3. (Color online) The SMZI could be used as a switch. The top switch is on and thus allows for the teleportation of Alice's qubit to Bob's qubit in channel A by means of the single photon produced at clock time $t$. However, the other switches are shown to be off and therefore the single photons produced from the sources aligned with these switches at the same time $t$ are unable to pass through channels $\mathrm{B}$ and $\mathrm{C}$ and carry on the transfer of quantum information. each arm by $-90^{\circ}$ pointing in the $-y$ direction (in plane) after a specified interaction time, at which point the photon is released back into the waveguide [weak-coupling regime (WCR)]. The photon is then able to self-interfere constructively and has its electric field positioned in the $-y$ direction. A second possible on configuration would involve the excess electron in the QDs having their spin parallel in the $+z$ direction (or up position), rather than the $-z$ direction as previously described, resulting again in constructive interference but with the final linear polarization state of the photon in the $+y$ direction. Conversely, the off configuration requires the spins of the excess electrons to be antiparallel, one spin being initialized in the down position $(-z$ direction) and the other in the up position ( $+z$ direction). This is depicted in the lower portion of Fig. 2. The polarization in the arm with the spin down $(\downarrow)$ electron is rotated by $-90^{\circ}$ and pointing in the $-y$ direction after a specified interaction time, while the polarization in the arm with the spin up $(\uparrow)$ is rotated by $+90^{\circ}$ and pointing in the $+y$ direction. The photon is then able to interfere destructively at the output of the SMZI.

We now consider the scheme in Fig. 3 that depicts how an array of SMZIs can be used to control the transfer of quantum information in parallel waveguides. Here the paths within the quantum network that have their SMZI switched off block the photon qubit from passing through, while those that have their SMZI switched on enable the transfer of quantum information by means of Greenberger-HorneZeilinger (GHZ) quantum teleportation (spin-photon-spin entanglement). ${ }^{9}$

Similarly, SMZIs can be used to achieve wavelength division multiplexing (MDM), without encoding, in quantum communication or quantum teleportation as shown in Fig. 4. In this case, the single-photon sources can be designed such that each photon qubit they emit has a different wavelength and therefore can share the same channel or fiber. This requires that each SMZI has its QD size and nanocavity size tuned such that its Faraday rotation rate and therefore its required interaction time remain the same as all the other interferometers independent of the wavelength at which they are being operated. This allows for a standardized technology in which all the components of the system can be synchronized.

\section{B. SMZI as a single-qubit gate}

Now we focus on how SMZIs can be used as a singlequbit gate for the photon or messenger qubit. In quantum networks, information is stored in the spin of the excess electron in QDs located at specific sites. Keeping track of sites using any physical addressing scheme, this information can be accessed and retrieved when needed. This information can then be transfer to another location (or QD) by means of a single photon that interacts with both the spins of the excess electrons at the QDs of origin and of destination creating a three particle entanglement or GHZ state resulting in teleportation. ${ }^{9}$

Single-qubit gates, as their name indicates, consist of operations involving only one qubit. Mathematically, a quantum gate acting on a single qubit can be described by a matrix, whereas the quantum state of the single qubit can be represented as a column vector. Because of the normalization 


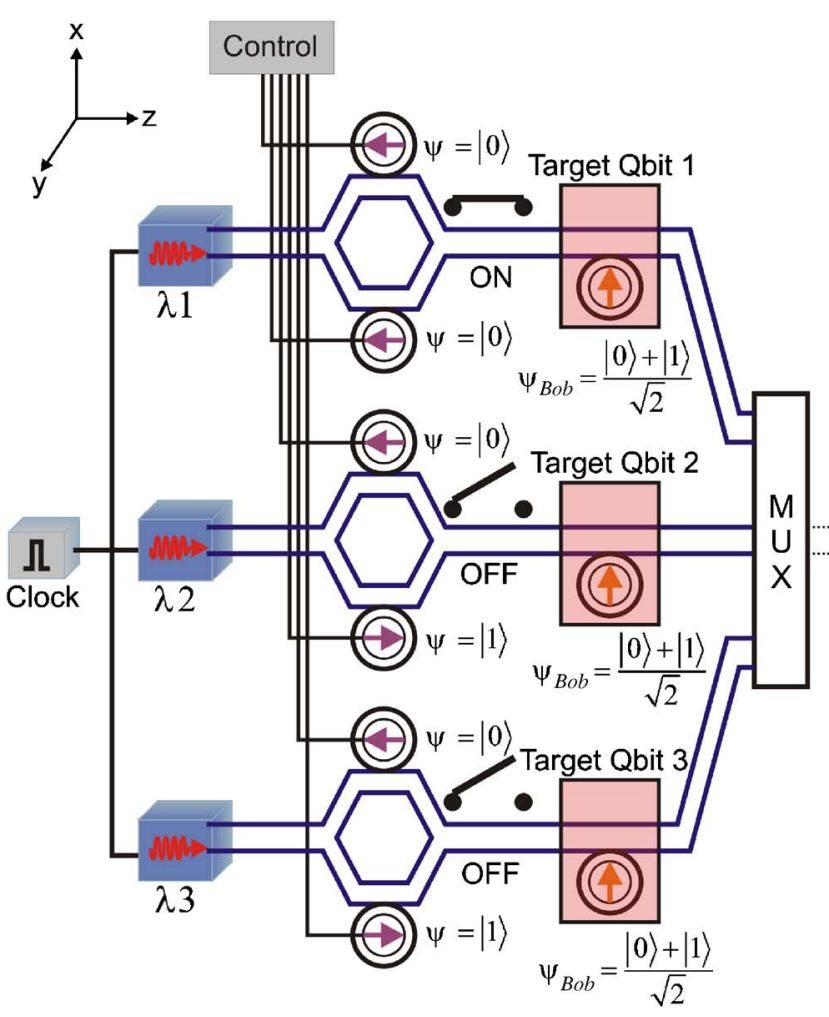

condition that requires $|\alpha|^{2}+|\beta|^{2}=1$ for the following quantum state $\alpha|0\rangle+\beta|1\rangle$, any matrix $U$ corresponding to a singlequbit gate must be unitary, that is, $U^{\dagger} U=I$,

$$
U \psi=\psi^{\prime} \rightarrow U\left[\begin{array}{l}
\alpha \\
\beta
\end{array}\right]=\left[\begin{array}{l}
\alpha^{\prime} \\
\beta^{\prime}
\end{array}\right] .
$$

Classically, there is only one nontrivial member of this class called the NOT gate, which transforms a 0 into a 1 and a 1 into a 0 . Similarly, a quantum gate that would transform the state $|0\rangle$ into the state $|1\rangle$, and vice versa, could be considered a quantum NOT gate. However, in quantum information processing, we are interested in states that are in a superposition of eigenbases. Due to this fact, unlike the classical case, there are technically an infinite number of nontrivial single-qubit gates. In this section, five important single-qubit gates known as the $X$ gate, the $Z$ gate, the $X Z$ gate, the Hadamard or $H$ gate, and the phase gate are considered. These are described by Eqs. (3)-(7). The $X, Z$, and $X Z$ gates are needed for the reconstruction of a state in the teleportation protocol. ${ }^{12}$ Note that our implementations of the $X, Z$, and $X Z$ gates by means of the SMZI have not been shown before. ${ }^{9}$ Our implementations are essential for the realization of a teleportation device that works only by means of the SPFE. Only then the teleportation method based on the SPFE can be completely integrated.

$$
\begin{array}{r}
\alpha|0\rangle+\beta|1\rangle \rightarrow \beta|0\rangle+\alpha|1\rangle, \\
\alpha|0\rangle+\beta|1\rangle \rightarrow \alpha|0\rangle-\beta|1\rangle, \\
\quad \text { XZ } \\
\alpha|0\rangle+\beta|1\rangle \rightarrow \beta|0\rangle-\alpha|1\rangle,
\end{array}
$$

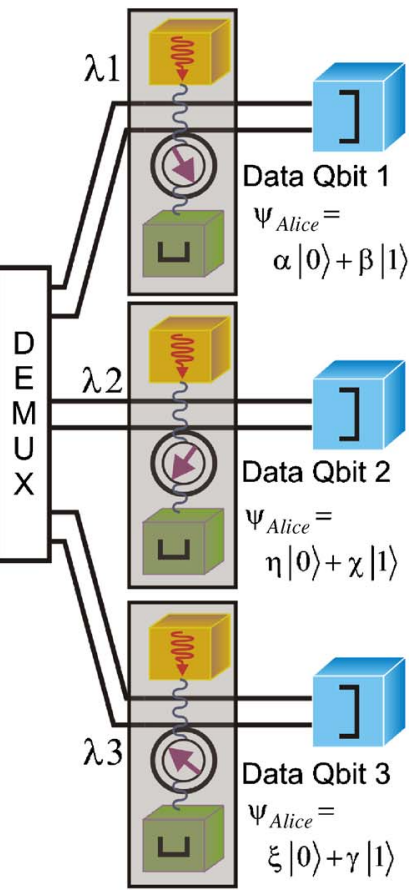

FIG. 4. (Color online) Switches based on the SMZI could also be implemented in a wavelength division multiplexing scheme for long distance quantum communication applications. Each single-photon source is tuned to a different wavelength; then they are entangled with their respective Bob's qubit before the multiplexing step. At the destination node, the single photons are demultiplexed before they are allowed to interact with their respective Alice's qubit to complete the teleportation process.

$$
\begin{aligned}
& \alpha|0\rangle+\beta|1\rangle \stackrel{H}{\rightarrow} \frac{\alpha+\beta}{\sqrt{2}}|0\rangle+\frac{\alpha-\beta}{\sqrt{2}}|1\rangle, \\
& \alpha|0\rangle+\beta|1\rangle \stackrel{R(\phi)}{\rightarrow} \alpha|0\rangle+\beta \cdot e^{2 \pi i \phi}|1\rangle,
\end{aligned}
$$

where

$$
\begin{aligned}
& X=\left[\begin{array}{ll}
0 & 1 \\
1 & 0
\end{array}\right], \quad Z=\left[\begin{array}{cc}
1 & 0 \\
0 & -1
\end{array}\right], \quad X Z=\left[\begin{array}{cc}
0 & -1 \\
1 & 0
\end{array}\right], \\
& H=\frac{X+Z}{\sqrt{2}}=\frac{1}{\sqrt{2}}\left[\begin{array}{cc}
1 & 1 \\
1 & -1
\end{array}\right], \quad \text { and } \quad R(\phi)=\left[\begin{array}{cc}
1 & 0 \\
0 & e^{2 \pi i \phi}
\end{array}\right] .
\end{aligned}
$$

In order to realize single quantum gates, a polarizing beam splitter is needed within the SMZI as opposed to the initially considered beam splitter that split the beam in two halves of equal amplitudes. Until a few years ago, most of the approaches in realizing polarizing beam splitters typically required relatively large size structures (length of the order of millimeters), which was undesirable for an integrated quantum technology. However, Kim et al. have proposed an ultracompact high-efficiency polarizing beam splitter that operates over a wide wavelength range and that is based on a hybrid photonic crystal and a conventional waveguide structure. ${ }^{13}$ Such technology could be implemented with the SMZI.

Figure 5 provides the configuration of the $X$ gate. We identify the logic qubits by $|0\rangle=|x\rangle,|1\rangle=|y\rangle$. Given the following general initial state for the photon qubit $\psi_{\text {photon }}$ $=\alpha|x\rangle+\beta|y\rangle$, the polarizing beam splitter splits the incoming photon into two components; $\alpha|x\rangle$ goes into the lower arm, while $\beta|y\rangle$ goes into the upper arm. Next, each polarization of the photon is rotated by $90^{\circ}$ in opposite direction so that it 


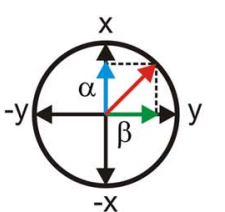

$\psi_{\text {photon }}=\alpha|x\rangle+\beta|y\rangle$
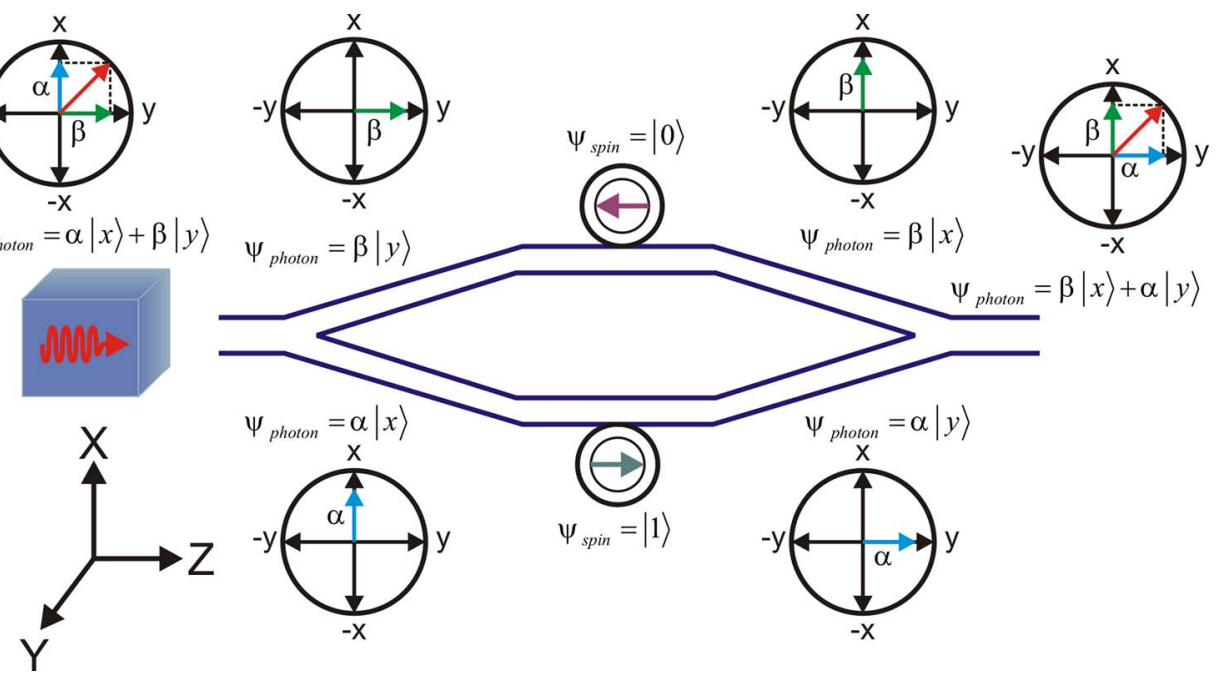
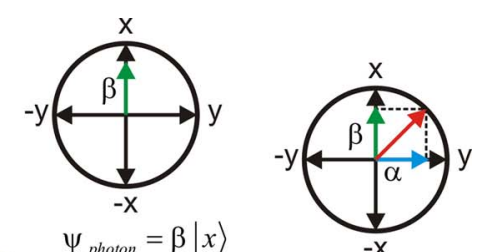

FIG. 5. (Color online) Configuration of the SMZI as a $X$ gate. corresponds to the other polarization eigenbasis (only two polarization eigenbasis in the given basis $|x\rangle$ and $|y\rangle)$ and yet retains the same amplitude. In other words, if the first quadrant is considered as depicted in Fig. 6, then the $x$ component of the polarization of the photon is rotated clockwise to $|y\rangle$, while the $y$ component is rotated counterclockwise to $|x\rangle$. After the different components of the photon polarization are recombined, the final state of the photon is $\psi_{\text {photon }}=\beta|0\rangle$ $+\alpha|1\rangle$. In respect to timing, the $X$ gate takes less than $100 \mathrm{ps}$ (see Sec. IV), which is approximately the interaction time needed to rotate both polarization components by $90^{\circ}$. Identical operations happen if the linear polarization of the single photon is within one of the other three possible quadrants.

A schematic of the $Z$ gate is shown in Fig. 7. Here we consider again that the general initial state for the photon qubit is $\psi_{\text {photon }}=\alpha|x\rangle+\beta|y\rangle$ and that the polarizing beam splitter splits the incoming photon into two components, $\alpha|x\rangle$ and $\beta|y\rangle$. Next, in the case of the $Z$ gate, only the polarization of the $y$ component is rotated by $180^{\circ}$. After the different components of the photon polarization are recombined, the final state of the photon is $\psi_{\text {photon }}=\alpha|x\rangle-\beta|y\rangle$. Similarly, the $Z$ gate takes less than 100 ps (see Sec. IV) to act on the photon qubit state.

A schematic of the $X Z$ gate is shown in Fig. 8. Here we consider again that the general initial state for the photon qubit is $\psi_{\text {photon }}=\alpha|x\rangle+\beta|y\rangle$ and that the polarizing beam splitter splits the incoming photon into two components, $\alpha|x\rangle$ and $\beta|y\rangle$. Next, in the case of the $Z$ gate, each polarization component of the photon is rotated by $90^{\circ}$ in the same counterclockwise direction (from the $+y$ direction to the $+x$ direction). After the different components of the photon polarization are recombined, the final state of the photon is $\psi_{\text {photon }}$ $=\beta|x\rangle-\alpha|y\rangle$. Similarly, the $X Z$ gate takes less than 100 ps (see Sec. IV) to act on the photon qubit state.

A schematic of the $H$ gate is depicted in Fig. 9. The photon qubit is again initialized to $\psi_{\text {photon }}=\alpha|x\rangle+\beta|y\rangle$, and the polarizing beam splitter splits the incoming photon into two components, $\alpha|x\rangle$ and $\beta|y\rangle$. Unlike the other gates, the $H$ gate requires two stages. In the first stage, each polarization component of the photon is rotated by $90^{\circ}$ in opposite directions. More precisely, the $X$ component of the polarization of the photon is rotated clockwise while the $Y$ component is rotated counterclockwise. In the second stage, each polarization component of the photon is rotated by only $45^{\circ}$ in the same counterclockwise direction. After the different components of the photon polarization are recombined, the final state of the photon is

$$
\psi_{\text {photon }}=\alpha \frac{|0\rangle+|1\rangle}{\sqrt{2}}+\beta \frac{|0\rangle-|1\rangle}{\sqrt{2}} .
$$
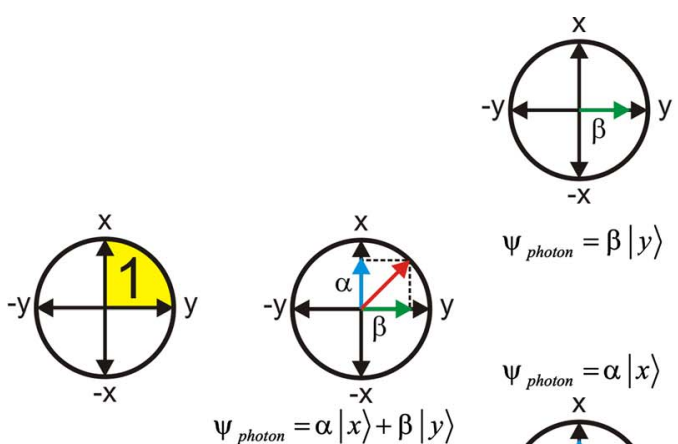
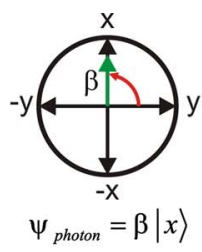
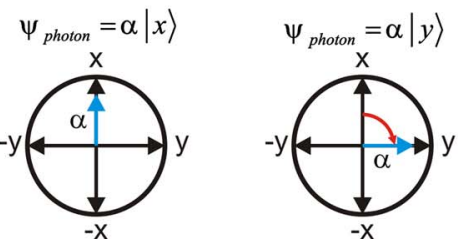

FIG. 6. (Color online) Diagram of $X$ gate operations on a single-photon polarization when the initial polarization is in the first quadrant.

$$
\psi_{\text {photon }}=\beta|x\rangle+\alpha|y\rangle
$$




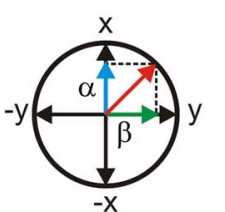

$\psi_{\text {photon }}=\alpha|x\rangle+\beta|y\rangle$
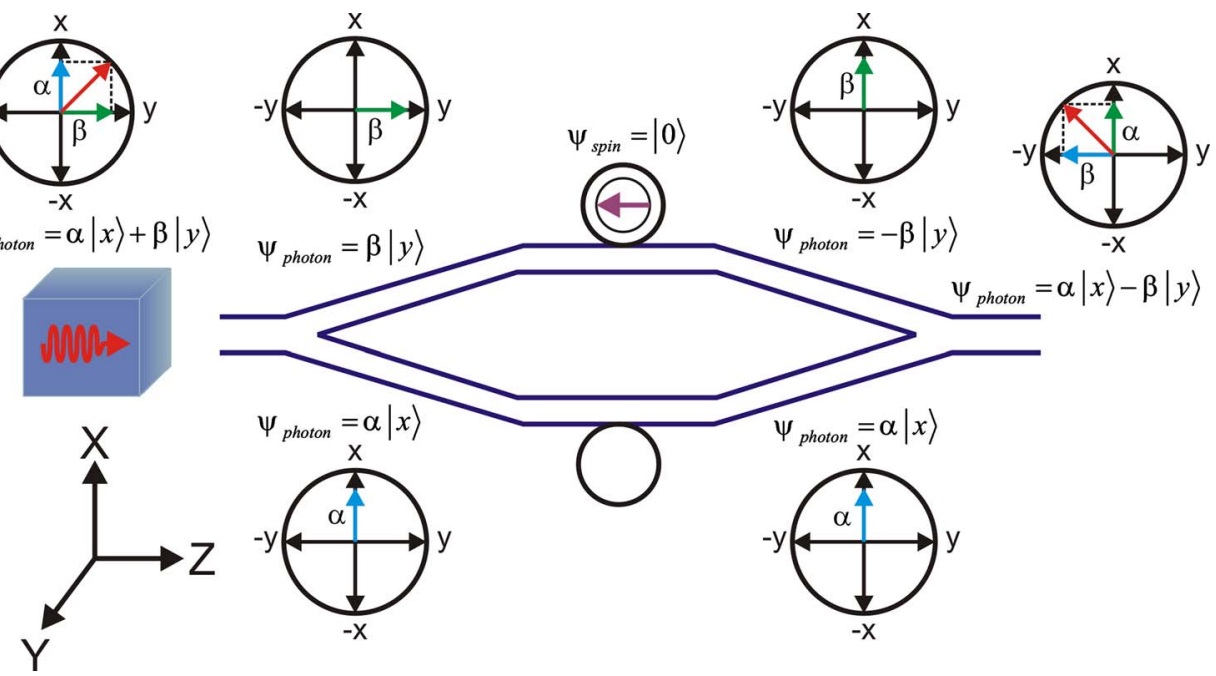

FIG. 7. (Color online) Configuration of the SMZI as a $Z$ gate.
Last, the phase gate is shown in Fig. 10. The $x$ and $y$ polarizations are converted into right and left circular polarizations by means of $\lambda / 4$ elements (quarter wave retardation plates), which further splits the $x$ and $y$ polarizations in two equal magnitudes within their respective arm before inducing a $\pi / 2$ phase resulting in LCP for the $x$ polarization and RCP for the $y$ polarization. The single spin leads then to a pure phase shift between the right and the left circular polarization, which can be converted back to $x$ and $y$ polarizations by means of more $\lambda / 4$ elements. Again, the entire operation takes less than 100 ps (see Sec. IV).

\section{DESIGN AND PERFORMANCE}

\section{A. Design}

For a feasible realization of such switches and gates, a traveling photon propagating within a waveguide must easily couple in an adjacent nanocavity; in which it must remain trapped for a specific interaction time before it is released in a way that minimizes the phase error back into the waveguide. There potentially exist several approaches to implement such devices. One possible scheme utilizes extremely high $Q$ cavities, which are assumed to be large enough to prevent the SCR. Whether or not this weak-coupling approach to the SPFE is desirable remains to be investigated, mainly because of the use of large and extremely high $Q$ cavities in providing an environment that favors short interaction times provides a challenge. In this publication, an alternate approach that is more suitable for large scale integration is proposed. This method is attractive in a large scale integration setting because its aim is to employ smaller cavity sizes, faster rates for the rotation of the linear polarization, and smaller $Q$ 's. This method is called the strong coupling approach to the SPFE, where the photon is sent in at one of the cavity polaritons.

The strong coupling approach to the SPFE requires that a photon strongly interacts with the QD (two-level system) inside the cavity, resulting in its linear polarization undergoing a faster conditional rotation consistent with the spin state of the excess electron in the conduction band. Such interaction calls for the SCR, which requires that the coupling constant $g$ between the QD and the cavity mode to be much larger than both the dipole dephasing rate $\gamma$ and the decay rate of the cavity $\Gamma$ [i.e., $g \gg(\gamma, \Gamma)]$. Furthermore, for the photon to be released, $g$ needs to be smaller than $\gamma$ and $\Gamma$, which is generally accepted as the condition for the WCR. ${ }^{14}$ Naturally, for a given material system, cavity volume, and wavelength, both the coupling constant $g$ and the dipole dephasing rate $\gamma$ are set and cannot be changed during device

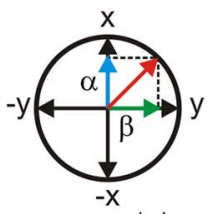

$\psi_{\text {photon }}=\alpha|x\rangle+\beta|y\rangle$
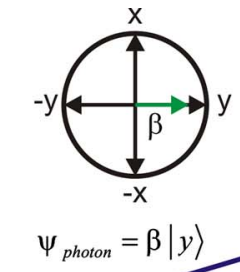

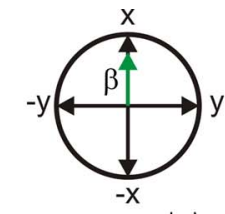

${ }^{-x}=\beta|x\rangle$

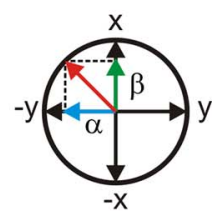

$$
=\beta|x\rangle-\alpha|y\rangle
$$

FIG. 8. (Color online) Configuration of the SMZI as a $X Z$ gate. 


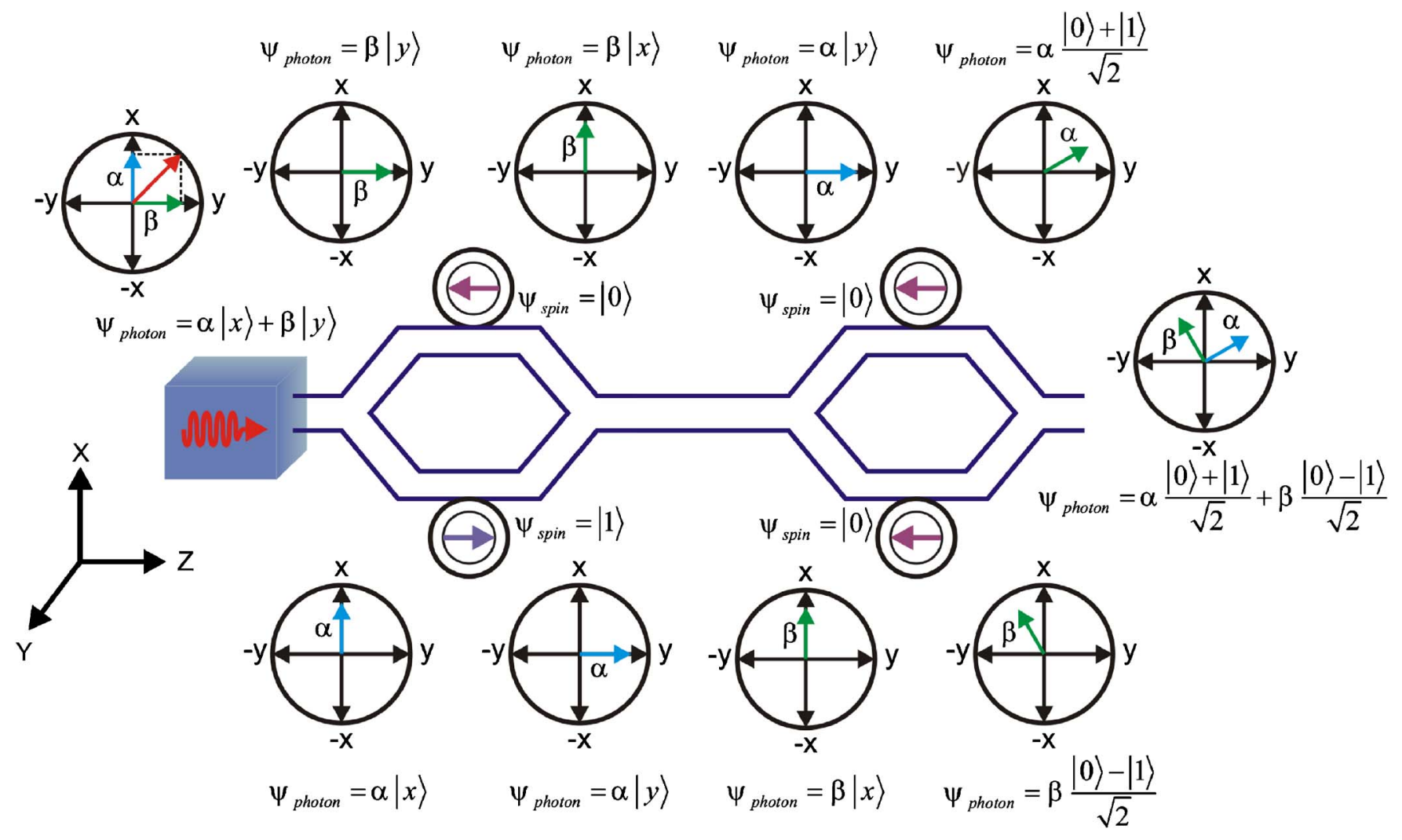

FIG. 9. (Color online) Configuration of the SMZI as a $H$ gate.

operation in a controllable way. Only the cavity decay rate $\Gamma$ is left in order to control the coupling regime in which the device is operating. Real-time lowering of the cavity decay rate (or increasing the cavity $Q$ ) promotes the SCR, while increasing of the cavity decay rate (or lowering the cavity $Q$ ) allows for the WCR. Therefore, by actively or passively controlling the $Q$ of the cavity, one can trap or release the photon at a given time.

For the physical realization of these SMZ based devices, the InAs/GaAs material system is considered. InAs selfassembled QDs are used to embody the two-level systems, which are embedded in high- $Q$ GaAs photonic-crystal nanocavities. Photonic crystals offer many potential advantages such as smaller cavity sizes (high electric field suitable for the SCR with a single QD), strong confinement (minimizing in-plane losses), and ultracompactness (sharp waveguide bend possible), thus providing the means for an integrated quantum technology. The ability to strongly confine light propagating in the plane within a two dimensional photoniccrystal structure is based on the existence of a photonic band gap due to a periodic change in the refractive index of the medium (optical modes whose frequencies are in the photonic band gap are unable to propagate within the photonic crystal). Because the SPFE requires the rotation of the linear polarization of the photon in a plane perpendicular to the direction of propagation, the photonic-crystal structure used to implement both the switch and the gates must support $x$ (out of plane) and $y$ (in plane) polarizations. As a result, the photonic-crystal structure must consist of a triangular lattice of air columns in GaAs, which has overlapping of both TE (electric field along $y$-axis or in-plane) and TM (electric field along $x$-axis or out-of-plane) photonic band gaps, as opposed to only one or the other for square lattices. ${ }^{15}$ Furthermore, the optical mode of interest is assumed to be far enough away from the band edges of both the TE and TM band gaps, since modes near band edges have smaller group velocities resulting in dramatic increase in $Q$ 's. For instance, modes closer to a TE band edges will bring about a higher $Q$ value for TE modes with respect to TM modes. Also, TM modes are subject to more in-plane radiation losses than TE modes, since triangular lattice photonic crystals suffer from a smaller TM band gap. In terms of phonic-crystal cavity $Q$ 's defined in Eq. (8), it means that the in-plane $Q\left(Q_{\perp}\right)$ for TM mode is smaller than the in-plane $Q$ for TE mode, while their out-ofplane $Q$ 's are approximately the same.

$$
Q=Q_{\|}+Q_{\perp} .
$$

The out-of-plane radiation losses can be eliminated so that $Q$ 's for both TE and TM modes are matched by (1) tailoring the mode profile, (2) making use of heterostructures, ${ }^{16}$ or (3) changing the shape of the scatterer (i.e., column or hole) in order to maximize the TM band gap. Finally, TE dipole modes of $L 1$ cavities are degenerate in theory (the $y$-polarized mode and the $z$-polarized mode), however, because of imperfections due to fabrication these optical modes split into different eigenenergies. Nevertheless, the resulting splitting is very small and the $Q$ 's for each mode do not vary significantly and can be considered the same; therefore, as long as the photon propagates in the plane ( $y$ or $z$ direction), the scheme remains unaffected. ${ }^{17}$

Before the cavity $Q$ 's and the cavity-waveguide coupling constant can be solved for, the dynamics of the two-level 


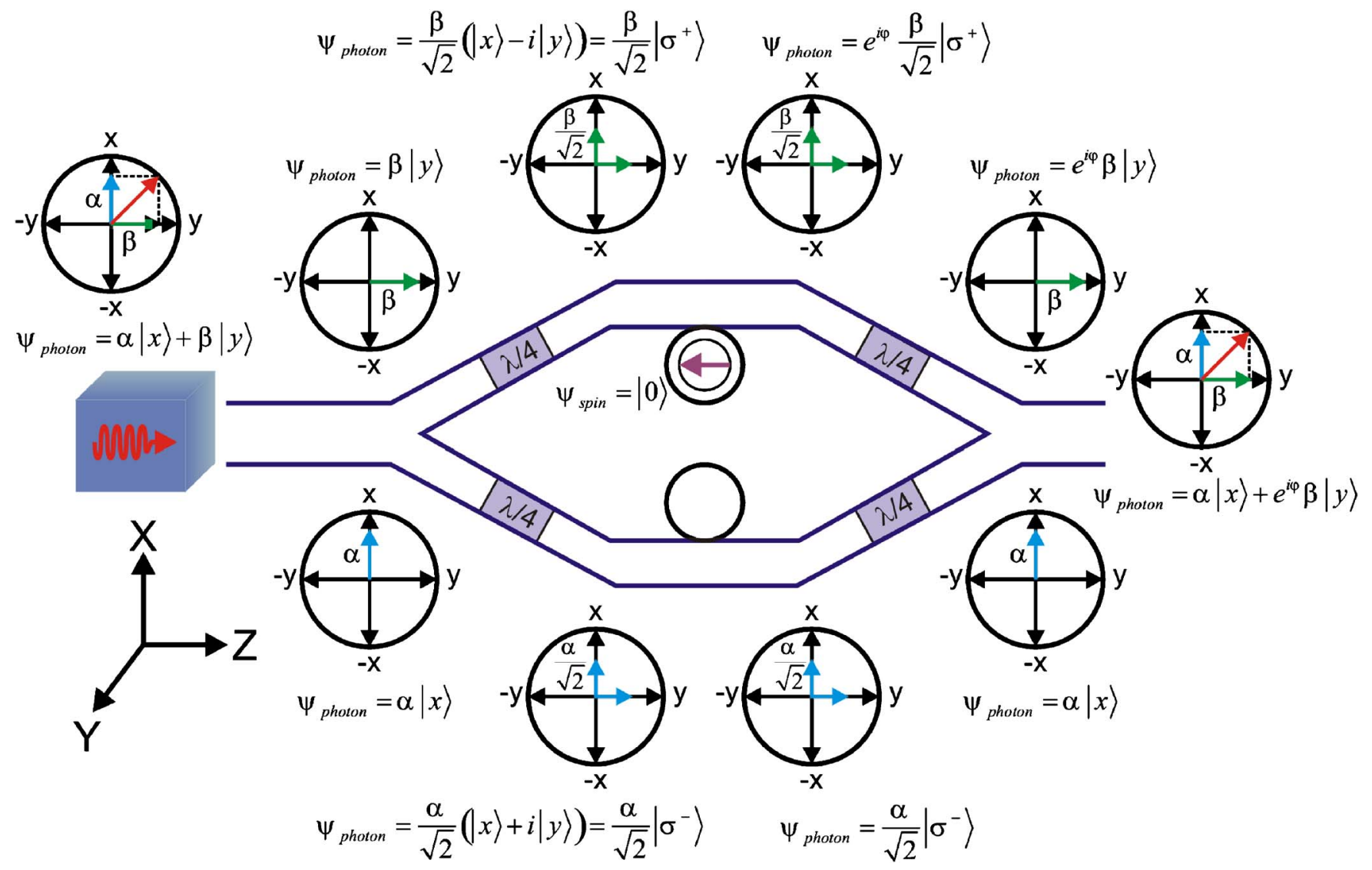

FIG. 10. (Color online) Configuration of the SMZI as a phase gate.

system (QD) interacting with a single mode quantum field (photon) must be investigated and the rate of rotation of the linear polarization determined. The Hamiltonian given in Eq. (1) describes how the RCP and LCP components of a linearly polarized photon field interact with the degenerate QD levels in a cavity. For simplicity, it is assumed that the spin of the excess electron in the conduction band is up $(\uparrow)$. At any time $t$, the state vector $|\psi(t)\rangle$ is a linear combination of the states $|\uparrow, \mathrm{hh}\rangle,|\uparrow, \mathrm{lh}\rangle,\left|\uparrow, \sigma_{z}^{+}\right\rangle$, and $\left|\uparrow, \sigma_{z}^{-}\right\rangle$. Here, $|\uparrow, \mathrm{hh}\rangle$ and $|\uparrow, \mathrm{lh}\rangle$ are the states in which the QD is in an excited state with a heavy hole (hh) exciton or a light hole (lh) exciton and the spin of the excess electron in the conduction band is up. $\left|\uparrow, \sigma_{z}^{+}\right\rangle$and $\left|\uparrow, \sigma_{z}^{-}\right\rangle$are the states in which the QD dot is in the ground state with the photon present in the cavity, and the spin of the excess electron in the conduction band is up. The state vector is therefore

$$
\begin{aligned}
|\psi(t)\rangle= & \frac{1}{\sqrt{2}}\left\{\left[C_{\uparrow h h}(t)|\uparrow, h h\rangle+C_{\uparrow \sigma^{+}}(t)\left|\uparrow, \sigma_{z}^{+}\right\rangle\right]\right. \\
& \left.+\left[C_{\uparrow l h}(t)|\uparrow, l h\rangle+C_{\uparrow \sigma-}(t)\left|\uparrow, \sigma_{z}^{-}\right\rangle\right]\right\} .
\end{aligned}
$$

The equations of motion for the probability amplitudes $C_{\uparrow h h}(t), C_{\uparrow l h}(t), C_{\uparrow \sigma^{+}}(t)$, and $C_{\uparrow \sigma^{-}}(t)$ can easily be derived and then solved exactly subject to certain initial conditions. The expressions for the probability amplitudes are

$$
\begin{aligned}
& C_{\uparrow h h}(t)=\left\{\left[\cos \left(\frac{\Omega_{3 / 2} t}{2}\right)-\frac{i \Delta}{\Omega_{3 / 2}} \sin \left(\frac{\Omega_{3 / 2} t}{2}\right)\right] C_{\uparrow h h}(0)\right. \\
& \left.-\left[\frac{i 2 g_{3 / 2 v, 1 / 2 c}}{\Omega_{3 / 2}} \sin \left(\frac{\Omega_{3 / 2} t}{2}\right)\right] C_{\uparrow \sigma^{+}}(0)\right\} e^{-i \cdot \Delta \cdot t / 2}, \\
& C_{\uparrow \sigma^{+}}(t)=\left\{\left[\cos \left(\frac{\Omega_{3 / 2} t}{2}\right)+\frac{i \Delta}{\Omega_{3 / 2}} \sin \left(\frac{\Omega_{3 / 2} t}{2}\right)\right] C_{\uparrow \sigma^{+}}(0)\right. \\
& \left.-\left[\frac{i 2 g_{3 / 2 v, 1 / 2 c}}{\Omega_{3 / 2}} \sin \left(\frac{\Omega_{3 / 2} t}{2}\right)\right] C_{\uparrow h h}(0)\right\} e^{-i \cdot \Delta \cdot t / 2}, \\
& C_{\uparrow l h}(t)=\left\{\left[\cos \left(\frac{\Omega_{1 / 2} t}{2}\right)-\frac{i \Delta}{\Omega_{1 / 2}} \sin \left(\frac{\Omega_{1 / 2} t}{2}\right)\right] C_{\uparrow l h}(0)\right. \\
& \left.-\left[\frac{i 2 g_{1 / 2 v, 1 / 2 c}}{\Omega_{1 / 2}} \sin \left(\frac{\Omega_{1 / 2} t}{2}\right)\right] C_{\uparrow \sigma^{-}}(0)\right\} e^{-i \cdot \Delta \cdot t / 2}, \\
& C_{\uparrow \sigma^{-}}(t)=\left\{\left[\cos \left(\frac{\Omega_{1 / 2} t}{2}\right)+\frac{i \Delta}{\Omega_{1 / 2}} \sin \left(\frac{\Omega_{1 / 2} t}{2}\right)\right] C_{\uparrow \sigma^{-}}(0)\right. \\
& \left.-\left[\frac{i 2 g_{1 / 2 v, 1 / 2 c}}{\Omega_{1 / 2}} \sin \left(\frac{\Omega_{1 / 2} t}{2}\right)\right] C_{\uparrow l h}(0)\right\} e^{-i \cdot \Delta \cdot t / 2},
\end{aligned}
$$

where $\Omega_{3 / 2}^{2}=\Delta^{2}+4 g_{3 / 2 v, 1 / 2 c}^{2}$ with $g_{3 / 2 v, 1 / 2 c}$ being the coupling 
strength involving a heavy hole electron, $\Omega_{1 / 2}^{2}=\Delta^{2}$ $+4 g_{1 / 2 v, 1 / 2 c}^{2}$ with $g_{1 / 2 v, 1 / 2 c}$ being the coupling strength involving a light hole electron, with $g_{3 / 2 v, 1 / 2 c}=\sqrt{3} g_{1 / 2 v, 1 / 2 c}$, and $\Delta$ $=\omega-\omega_{p h}$ being the detuning frequency. The rate of rotation of the linear polarization is proportional to the difference phase accumulated for the RCP and LCP components of the linear polarization during interaction with the QD with the frequencies $\Omega_{3 / 2}$ and $\Omega_{1 / 2}$, respectively. The coupling constants $g$ between the QD and the photon field can easily be obtained for GaAs. In order to carry out this calculation, the preferred cavity for the realization of the switch and the gates is the $L 1$ cavity (a single point defect). They do not necessarily possess $Q$ 's as high as $L 3$ cavities (three point defects along the $\Gamma-K$ direction), however, they usually provide the smallest mode volume. ${ }^{18}$ Shirane et al. ${ }^{17}$ reported a single defect or $L 1$ nanocavities based on a triangular lattice GaAs photonic-crystal membrane with mode volume of $V$ $=0.039 \mu \mathrm{m}^{3}$ and a $Q$ of17 000. Additionally, InAs QDs less than $25 \mathrm{~nm}$ in size are reported to have dipole moment $\mu_{h h}$ $=29$ (in Debye) with band gap energy $E_{g}=1.32 \mathrm{eV}$, which correspond to an emission wavelength of $\lambda=1.182 \mu \mathrm{m} .^{14}$

If we consider the configuration of the switch in Fig. 2, the single-photon qubit is initially linearly polarized along the $x$ axis or $\hat{e}_{x}=|x\rangle$. The expression for this linearly polarized photon can be rewritten in a different basis; one in which the linear polarization is considered as a superposition of right and left circular polarization,

$$
\begin{aligned}
\vec{e} & =|x\rangle=\frac{1}{\sqrt{2}}\left[\left(\frac{|x\rangle+i|y\rangle}{\sqrt{2}}\right)+\left(\frac{|x\rangle-i|y\rangle}{\sqrt{2}}\right)\right] \\
& =\frac{1}{\sqrt{2}}\left(\left|\sigma_{z}^{+}\right\rangle+\left|\sigma_{z}^{-}\right\rangle\right) .
\end{aligned}
$$

Once the photon interacts with the QDs in the separate arms of the SMZI, the right and left circular polarization components of the linear polarized photon accumulate different geometrical phases due to the single-photon Faraday rotation effect. Assuming that the spin of excess electron in the conduction band of the QD is up ( $\uparrow)$,

$$
\vec{e}=\frac{\left(e^{-i S_{O}^{\mathrm{hh}}}\left|\sigma_{z}^{+}\right\rangle+e^{i S_{O}^{\mathrm{lh}}}\left|\sigma_{z}^{-}\right\rangle\right)}{\sqrt{2}},
$$

where $S_{O}^{\mathrm{hh}}$ and $S_{O}^{\mathrm{lh}}$ represent the phase shift for the photon when interacting with the hh or lh, respectively. It is useful to rewrite the phase in order to be able to derive an expression for the effective angle $\varphi$, by which the linear polarization is rotated,

$$
\vec{e}=e^{-i \theta} \frac{\left(e^{-i \varphi}\left|\sigma_{z}^{+}\right\rangle+e^{i \varphi}\left|\sigma_{z}^{-}\right\rangle\right)}{\sqrt{2}},
$$

where

$$
\theta=\frac{\left(s_{O}^{\mathrm{hh}}+s_{O}^{\mathrm{lh}}\right)}{2}
$$

and

$$
\varphi=\frac{\left(s_{O}^{\mathrm{hh}}-s_{O}^{\mathrm{lh}}\right)}{2} .
$$

In terms of the linear polarization basis eigenstates, the polarization can be expressed as

$$
\vec{e}=e^{-i \theta}(\cos \varphi|x\rangle+\sin \varphi|y\rangle) .
$$

For the photon to destructively interfere with itself, the polarization initialized along the $x$ axis must be rotated $90^{\circ}$ in one arm so that it points in the $y$ direction (spin of excess electron is $\uparrow$ ) and $-90^{\circ}$ in the other arm so that it points in the $-y$ direction (spin of excess electron is $\downarrow$ ). This means that the angle $\varphi$ must be $\pi / 2$ in one arm and $-\pi / 2$ in the other arm. An expression for the phase shift accumulated for RCP and LCP components of the linearly polarized photon field during the interaction with the QD in the nanocavity can be derived from Eqs. (10)-(13). In order to solve for the phase accumulated for the RCP component $\left(S_{0}^{\text {hh }}\right)$, the time evolution of the probability $C_{\uparrow \sigma^{+}}(t)$ is used; whereas, the time evolution of the probability $C_{\uparrow \sigma^{-}}(t)$ is used for the phase accumulated for the LCP component $\left(S_{0}^{\mathrm{lh}}\right)$. Assuming the initial conditions are the following, $C_{\uparrow h h}(0)=0, C_{\uparrow \sigma^{+}}(0)=1$, $C_{\uparrow l h}(0)=0$, and $C_{\uparrow \sigma^{-}}(0)=1$, then the probability amplitudes of interest can be written as

$$
\begin{aligned}
C_{\uparrow \sigma^{+}}(t)= & e^{-i \cdot \Delta \cdot t / 2}\left[\cos \left(\frac{\Omega_{3 / 2} t}{2}\right)\right. \\
& \left.+\frac{i \Delta}{\Omega_{3 / 2}} \sin \left(\frac{\Omega_{3 / 2} t}{2}\right)\right] C_{\uparrow \sigma^{+}}(0), \\
C_{\uparrow \sigma^{-}}(t)= & e^{-i \cdot \Delta \cdot t / 2}\left[\cos \left(\frac{\Omega_{1 / 2} t}{2}\right)\right. \\
& \left.+\frac{i \Delta}{\Omega_{1 / 2}} \sin \left(\frac{\Omega_{1 / 2} t}{2}\right)\right] C_{\uparrow \sigma^{-}}(0) .
\end{aligned}
$$

Rewriting the complex coefficient $\left[\cos \left(\Omega_{3 / 2} t / 2\right)\right.$ $\left.+i \Delta / \Omega_{3 / 2} \sin \left(\Omega_{3 / 2} t / 2\right)\right]$ within the expression for the probability amplitude $C_{\uparrow \sigma^{+}}(t)$ in its exponential form using Euler's formula, an expression for the phase accumulated during the interaction of the RCP component with the hhs band can be obtained,

$$
S_{0}^{\text {hh }}=\tan ^{-1}\left[\frac{\Delta}{\Omega_{3 / 2}} \tan \left(\frac{\Omega_{3 / 2} t}{2}\right)\right] .
$$

Similarly, an expression for the phase accumulated during the interaction of the LCP component with the lhs band can be obtained,

$$
S_{0}^{\mathrm{lh}}=\tan ^{-1}\left[\frac{\Delta}{\Omega_{1 / 2}} \tan \left(\frac{\Omega_{1 / 2} t}{2}\right)\right] .
$$

Since the relative phase $\varphi=\left(S_{O}^{\mathrm{hh}}-S_{O}^{\mathrm{lh}}\right) / 2$ must be $\pm \pi / 2$, then $\left(S_{O}^{\mathrm{hh}}-S_{O}^{\mathrm{lh}}\right)$ must be equal to $\pi$. Moreover, because we do not want to produce an exciton ensuing the completion of the rotation of the linear polarization rotation, it must be required that $\Omega_{3 / 2} T / 2=j \pi$ and $\Omega_{1 / 2} T / 2=j \pi$ in Eqs. (10)-(13). It was found that a detuning energy of $E_{d}=75 \mu \mathrm{eV}$ gives an optimized operating point for both the phase requirement, $\varphi=\pi$ (see Fig. 11), and the probability amplitude requirements, $\Omega_{3 / 2} T / 2=j \pi$ and $\Omega_{1 / 2} T / 2=j \pi$ (see Fig. 12), resulting in an interaction time $T=43$ ps. Consequently, it can be said that the switching speed is on the order of tens of picosec- 


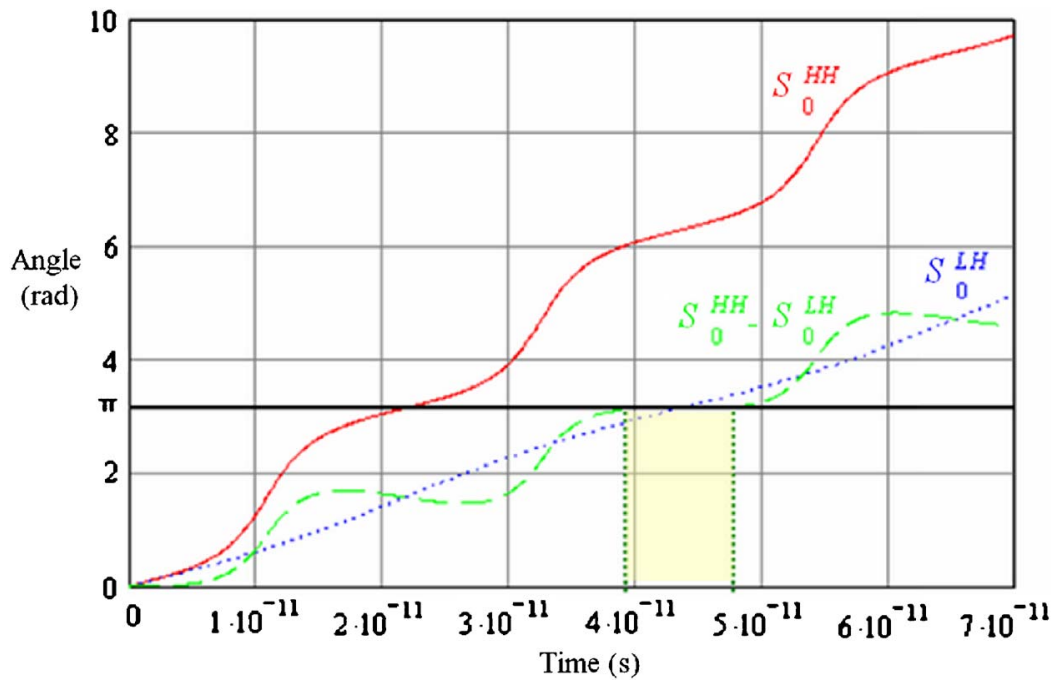

FIG. 11. (Color online) Phases accumulated during interaction as a function of time.

onds. Interestingly, the phase requirement is satisfied over a range of interaction times, which is from approximately $T$ $=39 \mathrm{ps}$ to $T=47 \mathrm{ps}$, while the probability amplitude requirements are satisfied exactly at $T=43$ ps with a very small variation within \pm 4 ps. This indicates that at this optimized operating point, this scheme is very robust against possible phase error within the considered range above. Furthermore, this interaction time is also much smaller than the limiting spin decoherence time of $20 \mathrm{~ms}$ in semiconductor nanostructures at a magnetic field of $4 \mathrm{~T}$ and at $1 \mathrm{~K}$ (Ref. 19) or $100 \mathrm{~ns}$ at zero magnetic field and $5 \mathrm{~K}^{20}$

Next, the coupling constants can be derived from the following equations:

$$
\begin{aligned}
& g_{3 / 2 v, 1 / 2 c}=\frac{\mu_{h h} E}{\hbar}, \\
& g_{1 / 2 v, 1 / 2 c}=\frac{\mu_{l h} E}{\hbar},
\end{aligned}
$$

where $E=\sqrt{\hbar \omega_{\mathrm{ph}} / 2 \varepsilon o \varepsilon r V}$ and $\mu_{h h}=\sqrt{3} \mu_{l h}$ This gives $g_{3 / 2 v, 1 / 2 c}=21 \mathrm{GHz}$ and $g_{1 / 2 v, 1 / 2 c}=7 \mathrm{GHz}$, which correspond to $\Omega_{3 / 2}=46 \mathrm{GHz}$ and $\Omega_{1 / 2}=23 \mathrm{GHz}$. The target cavity $Q$ 's and the cavity-waveguide coupling constant can also be de- termined. A commonly accepted condition for the SCR (Ref. 14) is

$$
2 g_{3 / 2 v, 1 / 2 c} \geq \frac{(\Gamma+\gamma)}{2},
$$

where $\Gamma$ is the cavity decay rate and $\gamma$ is the dipole dephasing rate. Equations for the cavity decay rate and the dipole dephasing rate are

$$
\begin{aligned}
& \Gamma=\frac{\omega_{\mathrm{ph}}}{Q}, \\
& \gamma=\gamma_{\mathrm{dot}}+\gamma_{\mathrm{enh}}=\gamma_{\mathrm{dot}}+F_{p} \gamma_{o}=\gamma_{\mathrm{dot}}+\left(\frac{3 Q \lambda^{3}}{4 \pi^{2} n^{3} V}\right) \gamma_{o},
\end{aligned}
$$

where $\gamma_{\mathrm{dot}}$ is the sum of the QD nonradiative dephasing rate and the radiative decay rate outside the cavity $\left(\gamma_{o}\right)$ and $\gamma_{\mathrm{enh}}$ is the enhanced rate due to the Purcell effect. As a consequence of Eq. (24), even a major source of optical dephasing in semiconductor QDs such as electron-phonon interaction usually resulting in the damping of Rabi oscillation can be ignored. $^{21}$ Therefore, for InAs/GaAs material system, the minimum $Q$ for which the condition in equation is satisfied is

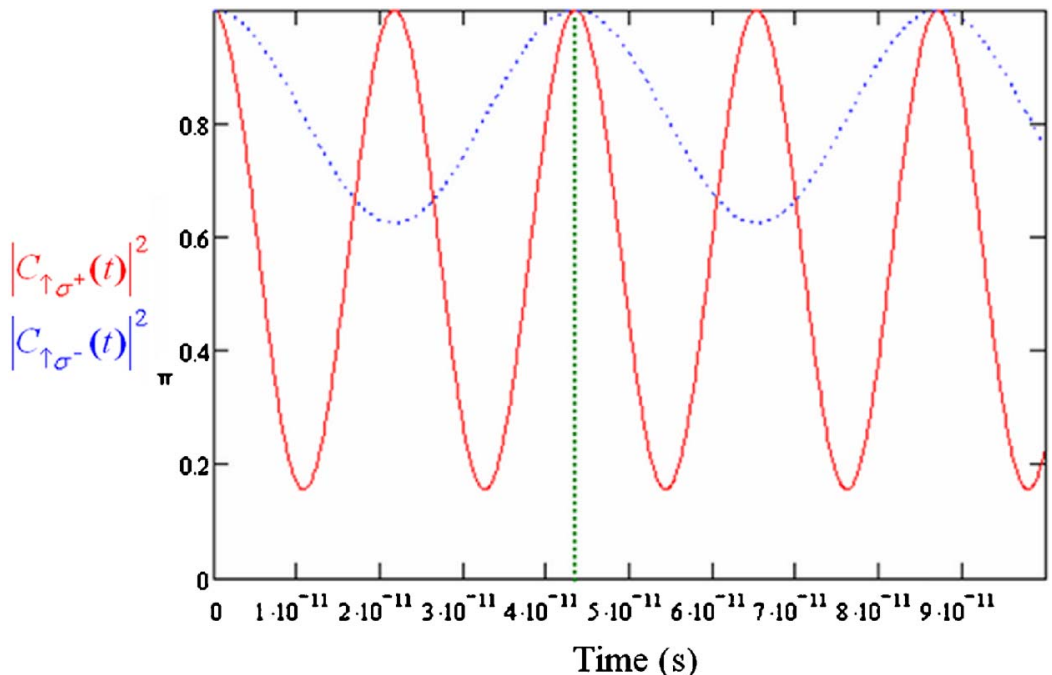

FIG. 12. (Color online) Time evolution of the probability amplitudes of left and right circular polarization components. 
approximately 6000 using $\gamma_{\mathrm{dot}}=22 \mathrm{GHz}, F_{p}=441$, and $\gamma_{o}$ $=0.088 \mathrm{GHz}^{14}$ Recalling that an optimized $L 1$ cavity can easily reach $Q$ 's of 17000 , the SCR is therefore achievable. If we assume that the cavity is designed to have a $Q$ of 12000 , well within the SCR, the associated cavity decay rate is $\Gamma=21 \mathrm{GHz}$. While this presents an ideal system for the SPFE, it represents a potential barrier for the release of the single photon back into the SMZI arm. Release of the single photon by the nanocavity requires a transition into the WCR by increasing $\Gamma$, thus necessitating a mechanism to control the $Q$ of the cavity in order to be able to release the photon back into the waveguide. Several methods can be envisioned to dynamically control the $Q$ of the cavity. Recently, control of a photonic-crystal cavity $Q$ factor was demonstrated by switching the cavity $Q$ from 12000 to 3000 in the picosecond regime using an optical pulse. ${ }^{22}$ Once the cavity $Q$ is switched to 3000 , the process of spontaneous emission is not reversible (i.e., in the WCR), resulting in an escape probability of $92 \%$ after a time $t=30$ ps using Eqs. (27) and (28). This alone provides much support for the feasibility of such devices.

$$
P(\%)=1-e^{-t / \tau},
$$

with

$$
\tau=\frac{1}{\Gamma}
$$

\section{B. Performance}

In order to estimate the fidelity, the error $\delta_{\varphi}$ in the phase $\varphi$ must be calculated [see Eqs. (16) and (17)]. These consist of the phase errors due to (1) the switching $\delta_{\varphi, Q \text { switch }}$, the escape time $\delta_{\varphi \text {,escape, }}$ and the excess electron spin state $\delta_{\varphi \text {,spin }}$, which are expressed in Eqs. (29)-(32). The phase error due to the switching can be obtained by calculating the ratio of the switching time over the interaction time. Phase error due to the escape time can be described in terms of the ratio of the rate of rotation of the linear polarization over the cavity decay rate. The rate of rotation is approximately the slope $m_{S^{l h}}$ of the phase accumulated during the interaction with the lhs $\left(S_{O}^{\mathrm{lh}}\right)$ in Fig. 11.

$$
\begin{aligned}
& \delta_{\varphi}=\delta_{Q \text { switch }}+\delta_{\varphi, \text { escape }}+\delta_{\varphi, \text { spin }}, \\
& \delta_{Q \text { switch }}=\frac{\Delta t_{Q \text { switch }}}{T}, \\
& \delta_{\varphi, \text { escape }} \approx \frac{m_{S^{l h}} / 2 \pi}{\Gamma}, \\
& \delta_{\varphi, \text { spin }}=\varphi_{\text {ideal }}-\varphi_{\text {actual }} .
\end{aligned}
$$

The phase error due to the initialization of the spin state is the difference in phase $\varphi$ accumulated between the ideal and actual spin states of the excess electron in the conduction band. Here, given the interaction time $T=43 \mathrm{ps}$, the time to switch the cavity $Q$ from 12000 to 3000 of $\Delta t_{Q \text { switch }}=4 \mathrm{ps},{ }^{22}$ the cavity decay rate of $\Gamma=84 \mathrm{GHz}$ at $Q=3000$, and the rate of rotation of the linear polarization $m_{S^{l h}} / 2 \pi=11.3 \mathrm{GHz}$. In addition, assuming that the initialization of the spin of the excess electron in the QD utilizes using a quantum optical control scheme based on the coupling of a trion (or charged exciton) state to two Zeeman-split spin states leading to an arbitrary rotation between the two spin states, ${ }^{23}$ it was found that such scheme would result in a total error of $0.01 \mathrm{rad}$ for a $\pi / 2$ rotation of the spin, ${ }^{24}$ which would correspond to the following spin state $\psi_{\text {spin }}=0.00005|\downarrow\rangle+0.99995|\uparrow\rangle$ instead of the initially assumed ideal spin state $\psi_{\text {spin }}=|\uparrow\rangle$. The state of the single-photon polarization after a 43 ps interaction is $\psi_{\text {photon }}=0.0052|x\rangle+0.9999|y\rangle$ using the ideal spin state $\left(\psi_{\text {spin }}=|\uparrow\rangle\right)$, which is equivalent to a rotation of $89.7^{\circ}$ (note that it is not $\psi_{\text {photon }}=|y\rangle$ because the interaction rates were rounded to the closest integer), and $\psi_{\text {photon }}=0.0052|x\rangle$ $+0.9999 \cdot e^{i \delta_{\theta, \operatorname{spin}}}|y\rangle$ with $\delta_{\theta \text {, spin }}=-8.1^{\circ}\left(\delta_{\theta \text {, spin }}\right.$ is the error in the general phase $\theta)$ using the actual spin state $\left(\psi_{\text {spin }}\right.$ $=0.00005|\downarrow\rangle+0.99995|\uparrow\rangle)$. Interestingly, the error in the spin state due to its initialization does not induce significant error in the phase $\varphi\left(\delta_{\varphi \text {,spin }} \simeq 0.00007\right)$, which determines the amount of rotation in the linear polarization (notice that the amplitudes for $|x\rangle$ and $|y\rangle$ components of the polarization remain the same). However, the error $\delta_{\theta \text {,spin }}$ in the general phase $\theta$ has a more significant consequence; it causes the $|x\rangle$ component of the polarization to be lagging the $|y\rangle$ component of the polarization possibly introducing some hellicity if the polarization following the rotation is not a pure $|y\rangle$ state. We will revisit $\delta_{\theta \text {,spin }}$ in more details later when we consider the extinction ratio and the insertion losses for the singlephoton switch device. Nonetheless, the following relative phase error is obtained $\delta_{\varphi}=\delta_{Q \text { switch }}+\delta_{\varphi \text {, escape }}+\delta_{\varphi \text {, spin }}=0.026$ $+0.1345+0.00007=0.16057\left(\delta_{\theta \text {,spin }}\right.$ can be ignored since it is much smaller than $\delta_{Q \text { switch }}$ and $\left.\delta_{\varphi \text {,escape }}\right)$.

There exists a quick solution to reduce the total phase error by simply reducing the cavity $Q$ to 10000 . Since the dynamic range $\Delta Q=9000$ is achievable, the cavity $Q$ at the moment of the switch will be 1000 . This corresponds to an escape probability of $92 \%$ after a time $t=10$ ps. The phase error is then $\delta_{\varphi}=0.026+0.044+0.00007 \cong 0.070$. This approach modifies only phase error due to the escape time.

Polarizers could in theory be used within each arm of the switch to eliminate the relative phase error $\delta_{\varphi}$. Consider the following general expression the $X$ and $Y$ components of the electric field vector:

$$
\vec{e}=e^{i \theta}\left[\cos (\varphi+\delta \phi)|x\rangle+\sin (\varphi+\delta \phi) e^{\left.i \delta_{\theta}|y\rangle\right]} .\right.
$$

Using the above calculated value of $\delta_{\varphi}=0.07$ and $\varphi=\pi / 2$ (the electric field is pointing in the $Y$ direction upon the completion of the SPFE), then $\varphi+\delta_{\phi}=1.64 \mathrm{rad}$. If the $Y$-polarizer is utilized, the resulting attenuation due to the relative phase error is approximately $0.48 \%$ of the intensity $\left(|\cos (\varphi+\delta \phi)|^{2}=0.004892\right)$, which corresponds to an efficiency of $99.51 \%$. This end result is significant and very promising for the realization of a quantum network based on this technology.

Furthermore, other losses are also traditionally small. Chen et al. $^{25}$ calculated propagation losses in photonic crystals and found a decaying constant $\beta=0.05 \mathrm{~cm}^{-1}$ for the fundamental guided mode (propagating in the plane) in a triangular lattice with air columns. This would correspond to an 
efficiency of $99.99 \%$ in terms of propagation losses for small structures such as the SMZI. (It is assumed that the probability for out-of-plane scattering is negligible). In addition, for the single-qubit gates, highly efficient polarizing beam splitters can be used. For instance, Seunghyun Kim et al. ${ }^{13}$ reported efficiencies above $99 \%$ for both TE and TM polarized lights propagating through their photonic-crystal based polarizing beam splitter.

Finally, the error $\delta_{\theta}$ in the general phase $\theta$ [see Eqs. (16) and (17)] and its effect on both the switch during the on and off stages, as well as the single-qubit gates, is investigated. The general phase can be understood as an effective path length difference between the two arms of the SMZI and cannot be corrected using a polarizer. It contributes to the insertion losses or attenuation when the switch is on; whereas, it is responsible for some optical leakage when the switch is off. Equations (34)-(37) below provide the expressions for the general phase error,

$$
\begin{aligned}
& \delta_{\theta}=\delta_{Q \text { switch }}+\delta_{\theta, \text { escape }}+\delta_{\theta, \text { spin }}, \\
& \delta_{Q \text { switch }}=\frac{\Delta t_{Q \text { switch }}}{T} \\
& \delta_{\theta, \text { escape }} \approx \frac{\left(m_{S h h}+m_{S l h}\right) / 2 \pi}{\Gamma} \quad\left(\Delta \simeq g_{3 / 2 v, 1 / 2 c}, g_{1 / 2 v, 1 / 2 c}\right), \\
& \delta_{\theta, \text { escape }}=\frac{\Omega_{3 / 2}+\Omega_{1 / 2}}{\Gamma} \quad\left(\Delta \gg g_{3 / 2 v, 1 / 2 c}, g_{1 / 2 v, 1 / 2 c}\right) \\
& \delta_{\theta, \text { spin }}=\theta_{\text {ideal }}-\theta_{\text {actual }} .
\end{aligned}
$$

If the cavity $Q$ at the time of the switch is assumed to be as low as 1000 , the general phase error is $\delta_{\theta}=0.026+0.15$ $+0.1413=0.3173$ for a detuning energy $E_{d}=75 \mu \mathrm{eV}$. Thus, if the switch is off, then the general phase with the error is $\theta=\pi+\delta_{\theta}=3.4588 \mathrm{rad}$ which means that $2.49 \%$ of the intensity goes through $[1+\cos (\theta) / 2=0.024959]$, corresponding to a $16 \mathrm{~dB}$ extinction ratio. On the other hand, if the switch is on $\left(\theta=0+\delta_{\theta}=0.3173 \mathrm{rad}\right), 2.49 \%$ of the intensity is lost on account of the error in the general phase, resulting in an efficiency of $97.5 \%$. This corresponds to an insertion loss of only $-0.109 \mathrm{~dB}$. This general phase error also causes the linear polarization of a photon qubit to become somewhat elliptical following a single-qubit operation. Such an effect can affect the fidelity of the single-qubit gate device or induce losses or leakage for the single-photon switch. The general phase error can be suppressed if a retardation element is added in each arm of the SMZI.

\section{CONCLUSION}

We have introduced a new concept for a SMZI that is based on the SPFE that can play a key role as a component for future quantum information devices or quantum networks. We demonstrated how the SMZI could be used as a switch to regulate the flow of quantum information both within a quantum computer and within a quantum commu- nication system using a WDM scheme. In addition, we introduced cases where SMZIs could also be used as single quantum gates such as the $X, Z, X Z, H$, and phase gates. In comparison with other proposed realizations of quantum computing (nuclear spin, harmonic oscillator, superconductors, etc.) this approach ranks second in terms of the maximum number of operations with $n_{\mathrm{op}}=10^{8}$ operations, which is defined as decoherence time $\left(10^{-3} \mathrm{~s}\right)$ over the interaction time $\left(10^{-12} \mathrm{~s}\right) .{ }^{19}$ The fidelity of the SMZI was investigated by considering the relative phase error due to switching and escape time, demonstrating the relative phase errors of only 0.07 rad which can be further reduced if polarizers are utilized. In addition, the general phase error though larger $(0.3173 \mathrm{rad})$ could be reduced using retardation elements placed in each arm of the SMZI.

\section{ACKNOWLEDGMENTS}

We would like to acknowledge the support for this work from the National Science Foundation under Grant No. ECCS-0725514.

${ }^{1}$ J. A. Wheeler and W. H. Zurek, Quantum Theory and Measurement (Princeton University Press, Princeton, NJ, 1983).

${ }^{2}$ M. O. Scully and K. Druhl, Phys. Rev. A 25, 2208 (1982).

${ }^{3}$ G.-Y. Xiang, J. Li, and G.-C. Guo, e-print arXiv:quant-ph/0607180

${ }^{4}$ G. Bertocchi, O. Alibart, D. B. Ostrowsky, S. Tanzilli, and P. Baldi, J. Phys. B 39, 1011 (2006)

${ }^{5}$ K. Shimizu and N. Imoto, Phys. Rev. A 62, 054303 (2000).

${ }^{6}$ E. Knill, R. Laflamme, and G. J. Milburn, Nature (London) 409, 46 (2001).

${ }^{7}$ M. A. Nielsen and I. Chuang, Quantum Computation and Quantum Information (Cambridge University Press, Cambridge, 2000).

${ }^{8}$ D. Englund, A. Faraon, B. Zhang, Y. Yamamoto, and J. Vučković, Opt. Express 15, 5550 (2007)

${ }^{9}$ M. N. Leuenberger, M. E. Flatte, and D. D. Awschalom, Phys. Rev. Lett. 94, 107401 (2005).

${ }^{10}$ S. A. Wolf, D. D. Awschalom, R. A. Buhrman, J. M. Daughton, S. von Molnar, M. L. Roukes, A. Y. Chtchelkanova, and D. M. Treger, Science 294, 1488 (2001).

${ }^{11}$ D. K. Young, J. A. Gupta, E. Johnston-Halperin, R. Epstein, Y. Kato, and D. D. Awschalom, Semicond. Sci. Technol. 17, 275 (2002).

${ }^{12}$ C. H. Bennett, G. Brassard, C. Crepeau, R. Jozsa, A. Peres, and W. K. Wootters, Phys. Rev. Lett. 70, 1895 (1993).

${ }^{13}$ S. Y. Kim, G. P. Nordin, J. B. Cai, and J. H. Jiang, Opt. Lett. 28, 2384 (2003).

${ }^{14}$ G. Khitrova, H. M. Gibbs, M. Kira, S. W. Koch, and A. Scherer, Nat. Phys. 2, 81 (2006).

${ }^{15}$ J. D. Joannopoulos, Photonic Crystals: Molding the Flow of Light (Princeton University Press, Princeton, 1995).

${ }^{16}$ T. Asano, B.-S. Song, Y. Akahane, and S. Noda, IEEE J. Sel. Top. Quantum Electron. 12, 1123 (2006).

${ }^{17}$ M. Shirane, S. Kono, J. Ushida, S. Ohkouchi, N. Ikeda, Y. Sugimoto, and A. Tomita, J. Appl. Phys. 101, 073107 (2007).

${ }^{18}$ L. C. Andreani, D. Gerace, and M. Agio, Phys. Status Solidi B 242, 2197 (2005).

${ }^{19}$ M. Kroutvar, Y. Ducommun, D. Heiss, M. Bichler, D. Schuh, G. Abstreiter, and J. J. Finley, Nature (London) 432, 81 (2004).

${ }^{20}$ J. M. Kikkawa and D. D. Awschalom, Phys. Rev. Lett. 80, 4313 (1998).

${ }^{21}$ J. Forstner, C. Weber, J. Danckwerts, and A. Knorr, Phys. Rev. Lett. 91, 127401 (2003).

${ }^{22}$ Y. Tanaka, J. Upham, T. Nagashima, T. Asano, T. Sugiya, and S. Noda, Nat. Mater. 6, 862 (2007).

${ }^{23}$ P. C. Chen, C. Piermarocchi, L. J. Sham, D. Gammon, and D. G. Steel, Phys. Rev. B 69, 075320 (2004)

${ }^{24}$ A. Grodecka, C. Weber, P. Machnikowski, and A. Knorr, Phys. Rev. B 76, 205305 (2007).

${ }^{25}$ Q. Chen, Y. Z. Huang, W. H. Guo, and L. J. Yu, Opt. Commun. 248, 309 (2005). 